\title{
Policies towards excellence in science and care giving with reference to Alzheimer-type dementia
}

\author{
A Belcastro \\ From de Senectute: Age and Health Forum \\ Catanzaro, Italy. 5-7 December 2009
}

In our country too, the growing number of elderly people together with the contemporary lowering of the birth rate, has now, and will continue to have in the near future, strong influence on choices to be made in healthcare and on welfare policy as a whole.

Therefore, the healthcare system must redefine its priorities and face a series of questions regarding the elderly. It will be necessary to rethink care giving functions taking into consideration the epidemiological revolution already underway, scientific progress and its practical applications in medicine together with the expectation for a better quality of life: we should be aiming at giving more life to years rather than more years to life.

So it is in this context that hospitals can no longer be seen as the only, prevalent answer to health and care needs. An entirely innovative network of prevention services, diagnosis, cure and rehabilitation for the non selfsufficient must be provided, innovative also in the way it is supplied and managed, able to respond to the requirements of the patient in an appropriate and punctual fashion.

Hospitalization of a patient suffering from dementia is always to be considered a critical event because of the protracted length of the stay, because of the increasing loss of vital functions in the patient together with the higher risk of complications including infection, falls and iatrogenic damage as well as an increased rate of mortality.

The network of services provided is, therefore, fundamental. Centres specialised in the diagnosis of dementia (Memory clinics, outpatient dementia clinics, Alzheimer assessment units) are able to enlarge upon traditional services by providing the following within the protocols

General Direction. Mater Domini University Hospital, Catanzaro, Italy of diagnostic assessment and specific care programs:

$\sim$ early identification of the illness

$\sim$ more accurate identification of illnesses responsi-

ble for dementia

$\sim$ improvement of quality of life of both patient and caregiver

$\sim$ more accurate treatment

Admission of dementia sufferers to special units has been shown to

$\sim$ reduce the frequency and intensity of behavioural disorders without, or with limited use of psychotropic drugs and physical constriction

$\sim$ delay functional loss

$\sim$ prevent complications including falls, under nourishment, immobility syndrome and delirium

$\sim$ improve the quality of the life of the patient, his family and caregivers

Special dementia units base their specific mission on four central pillars

$\sim$ the staff

$\sim$ the environment

$\sim$ the programs and activities

$\sim$ the family

Published: 19 May 2010

doi:10.1186/1471-2318-10-S1-L50

Cite this article as: Belcastro: Policies towards excellence in science and care giving with reference to Alzheimer-type dementia. BMC Geriatrics 2010 10(Suppl 1):L50. 